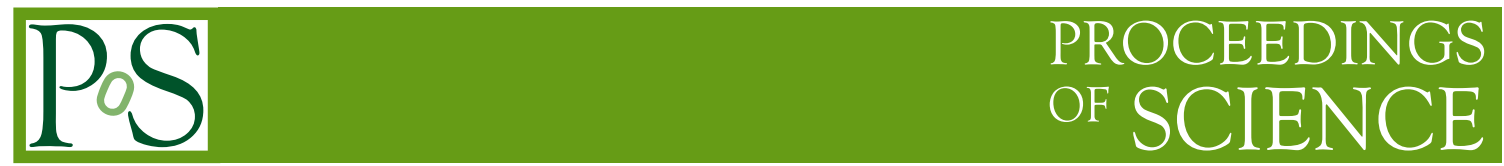

\title{
Unitarity Methods For One-Loop Amplitudes
}

\section{Simon Badger*}

Deutches Elektronen-Synchrotron DESY, Platanenallee, 6, D-15738 Zeuthen, Germany

E-mail: simon.badger@desy. de

We focus on new applications of generalised unitarity to analytic computations of one-loop helicity amplitudes with massive fermions. Working in the spinor/helicity formalism we compute compact analytic forms for the leading colour cut-constructible parts of $t \bar{t}$ production via gluon fusion at hadron colliders.

RADCOR 2009 - 9th International Symposium on Radiative Corrections (Applications of Quantum Field Theory to Phenomenology),

October 25 - 302009

Ascona, Switzerland

${ }^{*}$ Speaker. 


\section{Introduction}

Multi-particle interactions with massive particles will play an important role in new physics searches at the LHC. In order to uncover and distinguish new physics signals accurate predictions from NLO QCD corrections are essential. In these proceedings we outline recent progress in analytic computations of virtual corrections to one-loop amplitudes with massive fermions using newly developed on-shell tools.

New technologies for virtual amplitude calculations have opened up the possibilities of studying complicated multi-particle final states in hadron colliders. Numerical techniques have led the way giving the first NLO cross sections with four particle final states. QCD amplitudes with massive particles are computational more intensive due to the larger number of scales encountered. Nevertheless numerical implementations have been successful for $t \bar{t} b \bar{b}$ [1-3], $t \bar{t}+j$ [4]. Virtual corrections to $t \bar{t}+2 j$ have also been studied $[5,6]$ though at the present time the cross-section remains unknown.

Analytic computations may lead to a considerably faster evaluation of the cross-section allowing for more flexible phenomenological studies. A better understanding of the structure of the amplitudes would be beneficial to future computations and may help to improve speed and accuracy in future numerical algorithms.

\section{Generalised Unitarity}

Generalised unitarity has become an essential tool in the computation of multi-particle oneloop amplitudes over the last few years. Building from the pioneering work of Bern, Dixon, Dunbar and Kosower in the mid-nineties [7] the modern picture of unitarity gives a purely algebraic approach to the computations of one-loop amplitudes making use of complex analysis.

Quadruple cuts with complex momenta completely freeze the four dimensional loop integration leading directly to the algebraic evaluation of the associated scalar box integral coefficient [8]. The technique of integrand reduction first proposed by Ossola, Papadopoulos and Pittau [9] was shown to apply elegantly to the extraction of triangle and bubble coefficients using Laurent expansions of the unconstrained integrations by Forde [10]. This method has been generalised to the case of massive amplitudes [11] and is the basis for the method used here.

In these proceedings we apply the technique of generalised unitarity to compute compact analytic expression for one loop $t \bar{t} g g$ helicity amplitudes. The results are obtained from a semiautomated system using FORM and Maple. The integral basis and integrands are generated with Maple before the tree amplitudes (from BCFW recursion relations $[12,13]$ ) are expanded using FORM to extract closed analytic forms for the integral coefficients. IR consistency equations have been verified and used to constrain the form of the amplitude and ensure a compact representation.

Massive amplitudes also contain wave-function renormalisation and tadpole terms which are proportional to logarithms of the mass scales, $\log \left(\mathrm{m}^{2}\right)$. Such terms may also be determined from the universal UV and IR properties which is the technique we choose to employ here [14]. Firstly all bubble integrals are replaced with only their finite parts. The coefficients of the $\log \left(\mathrm{m}^{2}\right)$ and 
$\log \left(m_{H}^{2}\right)$ terms,

$$
C_{1 / \varepsilon} \frac{\Gamma(1+\varepsilon)}{\varepsilon}\left(\frac{\mu_{R}^{2}}{m^{2}}\right)^{\varepsilon}+C_{1 / \varepsilon}^{[H]} \frac{\Gamma(1+\varepsilon)}{\varepsilon}\left(\frac{\mu_{R}^{2}}{m_{H}^{2}}\right)^{\varepsilon},
$$

can then be read off by matching the universal IR poles [15] or the small mass expansion [16]. For the $t \bar{t} g g$ amplitudes considered here in the FDH scheme, after mass renormalisation, the missing information is simply,

$$
C_{1 / \varepsilon}=C_{F} A^{(0)}\left(1_{t}, 2,3,4_{\bar{t}}\right), \quad C_{1 / \varepsilon}^{[H]}=-\frac{4 N_{H}}{3 N} A^{(0)}\left(1_{t}, 2,3,4_{\bar{t}}\right) .
$$

where $N_{H}$ is the number of heavy flavours. For the time being we restrict ourselves to study only the cut-constructible parts of amplitudes.

\section{Spinor/Helicity Formalism}

For massless particles it is possible to completely decompose all momenta into a basis of two-component Weyl spinors since,

$$
p^{\mu}=\frac{1}{2}\left\langle p\left|\gamma^{\mu}\right| p\right]
$$

The polarisation vectors and fermion wave-functions then fit easily into a helicity basis,

$$
\begin{aligned}
u_{+}(p) & =|p\rangle & u_{-}(p) & =\mid p] \\
\varepsilon_{+}^{\mu}(p, \xi) & =\frac{\left\langle\xi\left|\gamma^{\mu}\right| p\right]}{\sqrt{2}\langle\xi p\rangle} & \varepsilon_{-}^{\mu}(p, \xi) & =\frac{\left\langle p\left|\gamma^{\mu}\right| \xi\right]}{\sqrt{2}[p \xi]}
\end{aligned}
$$

Kleiss and Stirling described how to construct well defined helicity states for massive momenta through the introduction of an arbitrary massless vector which defines the reference frame [17]. For a massive vector $P$ we use a massless vector $\eta_{P}$ to define a massless projected vector $P^{b}$ :

$$
P^{\mu}=P^{b, \mu}+\frac{m^{2}}{2 P \cdot \eta} \eta_{P}^{\mu}
$$

The $\bar{u}$ and $v$ spinors can then be defined by:

$$
\bar{u}_{ \pm}\left(P, m ; P^{b}, \eta_{P}\right)=\frac{\left\langle\eta_{P} \mp\right|(\not P+m)}{\left\langle\eta_{P} \mp \mid P^{b} \pm\right\rangle} \quad v_{ \pm}\left(P, m ; P^{b}, \eta_{P}\right)=\frac{(\not P-m)\left|\eta_{P} \pm\right\rangle}{\left\langle P^{b} \mp \mid \eta_{P} \pm\right\rangle}
$$

The freedom to keep $\eta_{P}$ arbitrary gives us the ability to relate the positive and negative helicity states by applying a simple transformation $\left(P^{b} \leftrightarrow \eta_{P}\right)$ :

$$
v_{-}\left(P, m ; P^{b}, \eta_{P}\right)=\frac{\left\langle P^{b} \eta_{P}\right\rangle}{m} v_{+}\left(P, m ; \eta_{P}, P^{b}\right) .
$$

It is therefore sufficient to consider helicity amplitudes where all massive fermion have positive helicity and obtain the remaining amplitudes by applying eq. (3.6). 


\section{Compact expressions for $t \bar{t} g g$ amplitudes}

In this section we present a short example for the techniques by applying them to the well studied case of top pair production through gluon fusion. The final results are obtained in an extremely compact analytic form and are in agreement with the results in the literature [18-20]. The amplitude is decomposed into colour ordered primitive amplitudes as outlined in [21]:

$$
\begin{aligned}
\mathscr{A}_{4}^{(0)}\left(1_{t}, 2,3,4_{\bar{t}}\right) & =\sum_{P(2,3)}\left(T^{a_{2}} T^{a_{3}}\right)_{i_{1} i_{4}} A_{4}^{(0)}\left(1_{t}, 2,3,4_{\bar{t}}\right) \\
\mathscr{A}_{4}^{(1)}\left(1_{t}, 2,3,4_{\bar{t}}\right) & =\sum_{P(2,3)} N\left(T^{a_{2}} T^{a_{3}}\right)_{i_{1} i_{4}} A_{4 ; 1}^{(1)}\left(1_{t}, 2,3,4_{\bar{t}}\right)+\delta^{a_{2} a_{3}} \delta_{i_{1} i_{4}} A_{4 ; 3}^{(1)}\left(1_{t}, 4_{\bar{t}} ; 2,3\right) \\
A_{4 ; 1}^{(1)}\left(1_{t}, 2,3,4_{\bar{t}}\right) & =A^{[L]}\left(1_{t}, 2,3,4_{\bar{t}}\right)-\frac{1}{N^{2}} A^{[R]}\left(1_{t}, 2,3,4_{\bar{t}}\right) \\
& +\frac{N_{f}}{N} A^{[f]}\left(1_{t}, 2,3,4_{\bar{t}}\right)+\frac{N_{H}}{N} A^{[H]}\left(1_{t}, 2,3,4_{\bar{t}}\right) \\
A_{4 ; 3}^{(1)}\left(1_{t}, 4_{\bar{t}} ; 2,3\right) & =\sum_{P(2,3)}\left\{A^{[L]}\left(1_{t}, 2,3,4_{\bar{t}}\right)+A^{[L]}\left(1_{t}, 2,4_{\bar{t}}, 3\right)+A^{[R]}\left(1_{t}, 2,3,4_{\bar{t}}\right)\right\} .
\end{aligned}
$$

There are two independent helicity amplitudes, ++++ and ++-+ . For simplicity we present only the leading colour amplitude for the "all-plus" helicity configuration as an example. All particles are considered to be out-going. The tree amplitude can be written compactly as:

$$
A_{4}^{(0)}\left(1_{t}^{+}, 2^{+}, 3^{+}, 4_{\bar{t}}^{+}\right)=-i m^{3} \frac{[23]\left\langle\eta_{1} \eta_{4}\right\rangle}{\langle 23\rangle\langle 2|1| 2]\left\langle\eta_{1} 1^{b}\right\rangle\left\langle\eta_{4} 4^{b}\right\rangle}
$$

The cut-constructible parts of the left-moving primitive amplitude is given by,

$$
\begin{aligned}
& -i A^{[L]}\left(1_{t}^{+}, 2^{+}, 3^{+}, 4_{\bar{t}}^{+}\right)=-\frac{\left\langle\eta_{1} \eta_{4}\right\rangle[32]^{2} m^{3}}{\left\langle\eta_{1} 1^{b}\right\rangle\left\langle\eta_{4} 4^{b}\right\rangle} I_{4}\left(s_{23}, s_{12}, 0,0, m^{2}, m^{2}, 0,0, m^{2}, 0\right) \\
& -\frac{\left(2 s_{12}\left\langle\eta_{1} \eta_{4}\right\rangle-\left\langle\eta_{1}\left|K_{12} K_{23}\right| \eta_{4}\right\rangle\right)[32] m^{3}}{\left\langle\eta_{1} 1^{b}\right\rangle\left\langle\eta_{4} 4^{b}\right\rangle\langle 23\rangle\langle 2|1| 2]^{2}} \widehat{I}_{2}\left(s_{12}, 0, m^{2}\right) \\
& -\frac{\Gamma(1+\varepsilon)}{2 \varepsilon}\left(\frac{\mu_{R}^{2}}{m^{2}}\right)^{\varepsilon} i A_{4}^{(0)}\left(1_{t}^{+}, 2^{+}, 3^{+}, 4_{\bar{t}}^{+}\right)+R_{4}^{[L]},
\end{aligned}
$$

where $\widehat{I}_{2}\left(s_{12}, 0, m^{2}\right)$ is the finite part of the scalar bubble integral and $R_{4}^{[L]}$ are the remaining rational terms. We have also defined $K_{i j}=p_{i}+p_{j}$ for convenience. The more complicated right-moving primitive amplitude is,

$$
\begin{aligned}
& -i A^{[R]}\left(1_{t}^{+}, 2^{+}, 3^{+}, 4_{\bar{t}}^{+}\right)=F_{4}\left(s_{23}, s_{12}, 0,0, m^{2}, m^{2}, m^{2}, m^{2}, 0, m^{2}\right) \\
& -\frac{\left\langle 2 \eta_{1}\right\rangle\left\langle 2 \eta_{4}\right\rangle\left(2 m^{2}+\langle 2|1| 2]\right)[32]^{2} m^{3}}{2\left\langle\eta_{1} 1^{b}\right\rangle\left\langle\eta_{4} 4^{b}\right\rangle\langle 23\rangle\langle 2|1| 3]}+\frac{\left\langle 3 \eta_{1}\right\rangle\left\langle 3 \eta_{4}\right\rangle\left(2 m^{2}+\langle 2|1| 2]\right)[32]^{2} m^{3}}{2\left\langle\eta_{1} 1^{b}\right\rangle\left\langle\eta_{4} 4^{b}\right\rangle\langle 23\rangle\langle 3|1| 2]} \\
& \left.-\frac{\left(2\left(2 m^{2}-s_{23}\right)\left\langle\eta_{1} \eta_{4}\right\rangle+2\left\langle\eta_{1}\left|K_{12} K_{23}\right| \eta_{4}\right\rangle\right)[32] m^{3}}{2\left\langle\eta_{1} 1^{b}\right\rangle\left\langle\eta_{4} 4^{b}\right\rangle\langle 23\rangle}\right)
\end{aligned}
$$




$$
\begin{aligned}
& +I_{3}\left(s_{23}, m^{2}, m^{2}, m^{2}, 0, m^{2}\right) \frac{\left(2 m^{2}-s_{23}\right)\left\langle\eta_{1} \eta_{4}\right\rangle[32] m^{3}}{\left\langle\eta_{1} 1^{b}\right\rangle\left\langle\eta_{4} 4^{b}\right\rangle\langle 23\rangle\langle 2|1| 2]}+I_{3}\left(s_{12}, 0, m^{2}, 0, m^{2}, m^{2}\right) \\
& \frac{\left(2\left\langle\eta_{1} \eta_{4}\right\rangle\langle 23\rangle+4\left\langle 2 \eta_{4}\right\rangle\left\langle 3 \eta_{1}\right\rangle\right)[32] m^{3}}{\left\langle\eta_{1} 1^{b}\right\rangle\left\langle\eta_{4} 4^{b}\right\rangle\langle 23\rangle^{2}}+\frac{\left\langle 3 \eta_{1}\right\rangle\left\langle 3 \eta_{4}\right\rangle\langle 2|1| 3][32] m^{3}}{\left\langle\eta_{1} 1^{b}\right\rangle\left\langle\eta_{4} 4^{b}\right\rangle\langle 23\rangle^{2}\langle 2|1| 2]}-\frac{\left\langle 2 \eta_{1}\right\rangle\left\langle 2 \eta_{4}\right\rangle\langle 3|1| 2][32] m^{3}}{\left\langle\eta_{1} 1^{b}\right\rangle\left\langle\eta_{4} 4^{b}\right\rangle\langle 23\rangle^{2}\langle 2|1| 2]} \\
& \left.+\frac{\left\langle 2 \eta_{1}\right\rangle\left\langle 2 \eta_{4}\right\rangle\langle 2|1| 2][32] m^{3}}{\left\langle\eta_{1} 1^{b}\right\rangle\left\langle\eta_{4} 4^{b}\right\rangle\langle 23\rangle^{2}\langle 2|1| 3]}-\frac{\left\langle 3 \eta_{1}\right\rangle\left\langle 3 \eta_{4}\right\rangle\langle 2|1| 2][32] m^{3}}{\left\langle\eta_{1} 1^{b}\right\rangle\left\langle\eta_{4} 4^{b}\right\rangle\langle 23\rangle^{2}\langle 3|1| 2]}\right)+I_{3}\left(0,0, s_{23}, m^{2}, m^{2}, m^{2}\right)( \\
& \left.\frac{\left\langle 2 \eta_{1}\right\rangle\left\langle 2 \eta_{4}\right\rangle[32]^{2} m^{3}}{2\left\langle\eta_{1} 1^{b}\right\rangle\left\langle\eta_{4} 4^{b}\right\rangle\langle 23\rangle\langle 2|1| 3]}-\frac{\left\langle 3 \eta_{1}\right\rangle\left\langle 3 \eta_{4}\right\rangle[32]^{2} m^{3}}{2\left\langle\eta_{1} 1^{b}\right\rangle\left\langle\eta_{4} 4^{b}\right\rangle\langle 23\rangle\langle 3|1| 2]}-\frac{\left\langle\eta_{1} \eta_{4}\right\rangle[32] m^{3}}{\left\langle\eta_{1} 1^{b}\right\rangle\left\langle\eta_{4} 4^{b}\right\rangle\langle 23\rangle}\right) \\
& -\widehat{I}_{2}\left(s_{12}, 0, m^{2}\right) \frac{\left(2 s_{12}\left\langle\eta_{1} \eta_{4}\right\rangle-\left\langle\eta_{1}\left|K_{12} K_{23}\right| \eta_{4}\right\rangle\right)[32] m^{3}}{\left\langle\eta_{1} 1^{b}\right\rangle\left\langle\eta_{4} 4^{b}\right\rangle\langle 23\rangle\langle 2|1| 2]^{2}} \\
& -\frac{\Gamma(1+\varepsilon)}{2 \varepsilon}\left(\frac{\mu_{R}^{2}}{m^{2}}\right)^{\varepsilon} i A_{4}^{(0)}\left(1_{t}^{+}, 2^{+}, 3^{+}, 4_{t}^{+}\right)+R_{4}^{[R]}
\end{aligned}
$$

where we define the finite integral combination:

$$
\begin{aligned}
& F_{4}\left(s_{23}, s_{12}, 0,0, m^{2}, m^{2}, m^{2}, m^{2}, 0, m^{2}\right)= \\
& \quad I_{4}\left(s_{23}, s_{12}, 0,0, m^{2}, m^{2}, m^{2}, m^{2}, 0, m^{2}\right)-\frac{1}{\langle 2|1| 2]} I_{3}\left(s_{23}, m^{2}, m^{2}, m^{2}, 0, m^{2}\right) .
\end{aligned}
$$

The contribution coming from heavy fermion loops is given by:

$$
\begin{aligned}
& -i A^{[H]}\left(1_{t}^{+}, 2^{+}, 3^{+}, 4_{\bar{t}}^{+}\right)=\frac{2 m m_{H}^{2}\left(\left\langle\eta_{1} \eta_{4}\right\rangle\langle 2|1| 2]+\left\langle 2 \eta_{1}\right\rangle\left\langle 3 \eta_{4}\right\rangle[32]\right)}{\left\langle\eta_{1} 1^{b}\right\rangle\left\langle\eta_{4} 4^{b}\right\rangle\langle 23\rangle^{3}[32]} \times \\
& \left(2 \widehat{I}_{2}\left(s_{23}, m_{H}^{2}, m_{H}^{2}\right)-s_{23} I_{3}\left(0,0, s_{23}, m_{H}^{2}, m_{H}^{2}, m_{H}^{2}\right)\right) \\
& +\frac{4 \Gamma(1+\varepsilon)}{3 \varepsilon}\left(\frac{\mu_{R}^{2}}{m_{H}^{2}}\right)^{\varepsilon} i A_{4}^{(0)}\left(1_{t}^{+}, 2^{+}, 3^{+}, 4_{\bar{t}}^{+}\right)+R_{4}^{[H]},
\end{aligned}
$$

from which we can read off the light fermion loop contribution:

$$
-i A^{[f]}\left(1_{t}^{+}, 2^{+}, 3^{+}, 4_{\bar{t}}^{+}\right)=R_{4}^{[f]}
$$

\section{Outlook}

We have shown that the generalised unitarity framework can be applied successfully to amplitudes with massive fermions to obtain compact analytic expressions. A semi-automated system for the computation of integral coefficients has been developed using FORM and Maple so that extension to processes with additional external partons is relatively straightforward. A detailed knowledge of the IR structure is essential and the cut-constructible parts of tadpole and wave-function renormalisation contributions have been fixed using the universal pole structure.

The obvious missing ingredients necessary to complete the amplitudes are the rational terms, $R_{4}^{[X]}$. It has already been demonstrated that these terms can be obtained numerically from $D$ dimensional generalised unitarity [5, 22]. In order to find the most compact representation it may also be useful to investigate the possibility of a dedicated Feynman reduction approach. 


\section{Acknowledgments}

I am indebted to David Kosower, Keith Ellis, Sven Moch, Christian Schwinn, Pierpaolo Mastrolia and Peter Uwer for many enlightening discussions. This work was supported by the Helmholtz Gemeinschaft under contract VH-NG-105.

\section{References}

[1] A. Bredenstein, A. Denner, S. Dittmaier and S. Pozzorini, JHEP 0808 (2008) 108 [arXiv:0807.1248 [hep-ph]].

[2] A. Bredenstein, A. Denner, S. Dittmaier and S. Pozzorini, Phys. Rev. Lett. 103 (2009) 012002 [arXiv:0905.0110 [hep-ph]].

[3] G. Bevilacqua, M. Czakon, C. G. Papadopoulos, R. Pittau and M. Worek, JHEP 0909, 109 (2009) [arXiv:0907.4723 [hep-ph]].

[4] S. Dittmaier, P. Uwer and S. Weinzierl, Phys. Rev. Lett. 98 (2007) 262002 [arXiv:hep-ph/0703120].

[5] R. K. Ellis, W. T. Giele, Z. Kunszt and K. Melnikov, Nucl. Phys. B 822, 270 (2009) [arXiv:0806.3467 [hep-ph]].

[6] A. van Hameren, C. G. Papadopoulos and R. Pittau, JHEP 0909 (2009) 106 [arXiv:0903.4665 [hep-ph]].

[7] Z. Bern, L. J. Dixon, D. C. Dunbar and D. A. Kosower, Nucl. Phys. B 425 (1994) 217 [arXiv:hep-ph/9403226].

[8] R. Britto, F. Cachazo and B. Feng, Nucl. Phys. B 725 (2005) 275 [arXiv:hep-th/0412103].

[9] G. Ossola, C. G. Papadopoulos and R. Pittau, Nucl. Phys. B 763 (2007) 147 [arXiv:hep-ph/0609007].

[10] D. Forde, Phys. Rev. D 75 (2007) 125019 [arXiv:0704.1835 [hep-ph]].

[11] W. B. Kilgore, arXiv:0711.5015 [hep-ph].

[12] R. Britto, F. Cachazo and B. Feng, Nucl. Phys. B 715, 499 (2005) [arXiv:hep-th/0412308].

[13] R. Britto, F. Cachazo, B. Feng and E. Witten, Phys. Rev. Lett. 94, 181602 (2005) [arXiv:hep-th/0501052].

[14] Z. Bern and A. G. Morgan, Nucl. Phys. B 467, 479 (1996) [arXiv:hep-ph/9511336].

[15] S. Catani, S. Dittmaier and Z. Trocsanyi, Phys. Lett. B 500 (2001) 149 [arXiv:hep-ph/0011222].

[16] A. Mitov and S. Moch, JHEP 0705, 001 (2007) [arXiv:hep-ph/0612149].

[17] R. Kleiss and W. J. Stirling, Nucl. Phys. B 262, 235 (1985).

[18] J. G. Korner, Z. Merebashvili and M. Rogal, Phys. Rev. D 73 (2006) 034030 [arXiv:hep-ph/0511264].

[19] C. Anastasiou and S. M. Aybat, Phys. Rev. D 78, 114006 (2008) [arXiv:0809.1355 [hep-ph]].

[20] S. D. Badger, Nucl. Phys. Proc. Suppl. 183 (2008) 220 [arXiv:0807.1245 [hep-ph]].

[21] Z. Bern, L. J. Dixon and D. A. Kosower, Nucl. Phys. B 437, 259 (1995) [arXiv:hep-ph/9409393].

[22] K. Melnikov and M. Schulze, JHEP 0908, 049 (2009) [arXiv:0907.3090 [hep-ph]]. 